\title{
Ratio Between Virtual and Visual Lesion Size as a Measure to Describe Reduction in Leaf Photosynthesis of Rice Due to Leaf Blast
}

\author{
L. Bastiaans
}

Department of Theoretical Production Ecology, Wageningen Agricultural University, P.O. Box 430, 6700 AK Wageningen, The Netherlands.

Thanks are due to C. Q. Torres, former postdoctoral scientist of the Department of Plant Pathology of the International Rice Research Institute (IRRI), Los Banos, The Philippines, for providing plant material for photosynthesis measurements. P. S. Teng and J. M. Bonman of the Department of Plant Pathology of IRRI are acknowledged for providing rice seeds and fungal isolates for the greenhouse experiment. Gratitude is extended to R. Rabbinge, J. C. Zadoks, M. J. Kropff, and W. v.d. Werf for critical reading of the manuscript.

Accepted for publication 23 October 1990 (submitted for electronic processing).

\section{ABSTRACT}

Bastiaans, L. 1991. Ratio between virtual and visual lesion size as a measure to describe reduction in leaf photosynthesis of rice due to leaf blast. Phytopathology 81:611-615.

The effect of Pyricularia oryzae, the causal organism of blast in rice, on net photosynthetic rate of rice leaves was measured in the field and in a greenhouse experiment. Leaf blast reduced photosynthesis not only through a reduction in green leaf area, but also through an effect on photosynthesis of the remaining green leaf tissue. A function was derived to relate the net photosynthetic rate of diseased leaf area $\left(P_{x}\right)$ to the photosynthesis of comparable healthy leaf area $\left(P_{O}\right)$ and disease severity $(x): P_{x}=P_{O}(1-x)^{\beta}$. This function is based on the assumption that the visual lesion is part of a virtual lesion in which photosynthesis is negligible. The parameter $\beta$ expresses the ratio between virtual and visual lesion size and characterizes the effect of the pathogen on leaf photosynthesis for the entire range of measured disease severities. A value of $\beta$ between 3 and 4 gave a good description of the effect of leaf blast on net photosynthetic rate of rice leaves, indicating a much stronger effect of leaf blast on leaf photosynthesis than expected on the basis of visible lesion size.

Additional keywords: Oryza sativa.

After infection of rice leaves with Pyricularia oryzae Cavara, the causal organism of blast in rice (Oryza sativa L.), ellipticalshaped lesions appear on leaves of susceptible cultivars. Goto (6) reported that yield loss due to leaf blast exceeded yield loss caused by cutting off of a percentage of leaf area equal to the percentage leaf covered by blast. This extra reduction meant that leaf blast influenced the host plant more than just the loss in leaf area. For various pathosystems, an effect of the pathogen on photosynthesis of the remaining green leaf area has been reported (for example, Erysiphe graminis on wheat [18] and Alternaria alternata on cotton [5]). Pathosystems in which the pathogen did not impair photosynthesis of healthy leaf tissue also have been reported (for example, Puccinia recondita on wheat [19] and Phytophthora infestans on potato [22]). Obviously, the effect of a pathogen on host photosynthesis varies according to the pathosystem under consideration.

As far as the effect of $P$. oryzae on photosynthesis of rice is concerned, only observations on canopy photosynthesis of seedlings have been reported. Burrell and Rees (3) and Padhi et al (16) observed a marked reduction in canopy photosynthesis of inoculated seedlings when compared with the performance of healthy plants. Whether the reduction was solely attributable to a reduction in green leaf area was not determined.

In this study, the effect of $P$. oryzae on leaf photosynthesis was measured, and a function was derived to describe the dependence of leaf photosynthetic rate on the fraction of leaf area with blast lesions.

\section{MATERIALS AND METHODS}

In an experimental field at the International Rice Research Institute, Los Banos, The Philippines, net rates of leaf photosynthesis were measured to determine the effect of leaf blast on photosynthesis of rice leaves. Similar observations were made

Cㄱ 1991 The American Phytopathological Society in Wageningen, The Netherlands, at the Centre for Agrobiological Research, using rice plants grown in a greenhouse.

Plant material in the field experiment. In the spring of 1988 , a field experiment was conducted to assess yield loss due to rice blast. Part of the plant material in this experiment was used for measurements of leaf photosynthesis. In the experiment, 11-dayold seedlings of $O$. sativa (cultivar IR50) were transplanted in a large plot $\left(2,500 \mathrm{~m}^{2}\right)$ at a planting distance of $0.2 \times 0.2 \mathrm{~m}$. Fertilizer (N-P-K, $60-50-50 \mathrm{~kg} / \mathrm{ha}$ ) was applied 2 days before transplanting. Plants were grown under irrigated conditions. Three days after transplanting, different densities of blast diseased seedlings were planted between rows of the rice seedlings. In this way, different levels of blast epidemics were generated in the field. A disease rating at maximum tillering revealed that diseased leaf area per hill, affected by blast, ranged from 0 to $50 \%$. At the same growth stage, rates of leaf photosynthesis were measured, using plants in randomly selected healthy and diseased parts of the experimental field. Fully developed leaves in the top layers of the canopy were selected for the determination of leaf photosynthesis. Because of the natural disease development, measured leaves contained lesions in different developmental stages.

Plant material in the greenhouse experiment. Rice plants (cultivar IR50) were grown in 21-cm-diameter closed pots filled with sand. Before sowing, seeds were kept in moist petri dishes for 5 days. Germinated seeds were selected, and five seeds were sown per pot. Plants were grown in a greenhouse during the summer of 1988. Measurements revealed that radiation inside was $70 \%$ of the radiation outside the greenhouse. Temperature ranged between $18 \mathrm{C}$ during the night and $30 \mathrm{C}$ in the daytime, and relative humidity (RH) varied between $60 \%$ (day) and $95 \%$ (night). Nitrogen fertilizer was added to the pot soils at a rate of $500 \mathrm{mg}$ of $\mathrm{NH}_{4} \mathrm{NO}_{3}$ per pot at 12 and 22 days after sowing. The pots were inundated by maintaining the water level at $0-1$ $\mathrm{cm}$ above the soil surface.

Plants were inoculated with $P$. oryzae at 27 days after sowing. The fungus was grown on prune agar at a temperature of $28 \mathrm{C}$. Inoculum was prepared as described by Mackill and Bonman 
(13), and the spore density was adjusted to $5 \times 10^{4}$ conidia $/ \mathrm{ml}$. Gelatin was added to the inoculum in a concentration of $2.5 \mathrm{~g} / \mathrm{L}$. Plants were sprayed with inoculum until runoff using a portable air compressor. Control plants were sprayed with a gelatin solution. All plants were incubated in a moist chamber for 36 hr at $23 \mathrm{C}$.

Symptoms appeared on leaves 3-4 days after inoculation, and new spores were produced after further lesion development. Because conditions in the greenhouse were favorable for infection, newly infected leaves with lesions in different developmental stages appeared. This closely resembled a situation of natural disease development in the field. At maximum tillering, $2.5 \mathrm{wk}$ after inoculation, photosynthesis on leaves of healthy and diseased plants was measured.

Photosynthesis measurements. In both experiments, net rates of photosynthesis were measured with a portable leaf chamber analyzer (Analytical Development Co., UK). The rate of photosynthesis was calculated following the procedure described by von Caemmerer and Farquhar (23). In the field experiment, photosynthesis was measured at light saturation. Average conditions within the chamber were as follows: $330 \mathrm{Jm}^{-2} \mathrm{~s}^{-1}$ of photosynthetically active radiation (PAR) $(400-700 \mathrm{~nm}$ ), temperature of $34 \mathrm{C}$, and $\mathrm{RH}$ of $63 \%$. In the greenhouse experiment, an incandescent lamp was used to reach a high incident radiation at a stable level. Average conditions within the chamber were as follows: $230 \mathrm{Jm}^{-2} \mathrm{~s}^{-1}$ of PAR, temperature of $31 \mathrm{C}$, and $\mathrm{RH}$ of $43 \%$.

In both experiments, fully developed leaves in the top layers of the canopy were selected. After photosynthesis was measured, the enclosed part of the measured leaf was harvested, and leaf width was determined. Total leaf surface was calculated by multiplying leaf width with the length of the leaf chamber $(5.6$ $\mathrm{cm})$. The lesions on this part of the leaf were traced on a plastic sheet with a fine black overhead marker. Total area of traced lesions was determined with a digital video image analyzer (Area Meter System 3439, DELTA-T Devices Ltd., England). Lesions of both chronic and acute lesion type (15) were observed. Chronic lesions are brown, whereas acute lesions have a grayish center and a brown margin and are sometimes surrounded by a yellow halo. For this study, a visible lesion was defined to consist of brown (chronic) or grayish, brown, and yellow (acute) leaf area. Small differences in intensity of the green color sometimes were observed around lesions of the acute type. However, leaf area of any green color was defined as not being part of a visible lesion.

Accordingly, disease severity was defined as the fraction of leaf area covered by visible lesions. In both experiments, disease severity on measured leaves ranged from 0 to 0.3 .

Model to relate disease severity and leaf photosynthetic rate. Justesen and Tammes (9) described a formula to estimate the fraction of leaf area remaining visibly healthy $(1-x)$ when both the number of lesions and the fraction of leaf area occupied by a single lesion are known. This formula takes into account the possible overlap of individual lesions:

$$
(1-x)=(1-\alpha)^{n}
$$

in which $x=$ visibly diseased fraction of the leaf area; $n=$ number of lesions (spots) per leaf; $\alpha=$ fraction of the leaf area occupied by a single lesion, which can be calculated as $A / M$, with $A=$ the area of a single lesion (spot) and $M=$ the area of a leaf.

An implicit assumption made in equation 1 is that the probability of infection on any part of the leaf is proportional to the area of this part, regardless of its position on the leaf and the presence of former infections. An identical formula is valid if the influence of a pathogen is not restricted to the visual lesion with area $A$ but to a larger area $A A$, which is called the virtual lesion. In that case the fraction of leaf area remaining virtually healthy $(1-y)$ can be estimated as:

$$
(1-y)=(1-\beta \alpha)^{n}
$$

in which $y=$ virtually diseased fraction of leaf area, and $\beta=$ $A A \mid A$, the ratio between the leaf area occupied by the virtual lesion and the leaf area occupied by the visual lesion. After rewriting equation 1 :

$$
n=\ln (1-x) / \ln (1-\alpha)
$$

and substituting into equation 2, a relation between $x$ and $(1-y)$ is obtained:

$$
(1-y)=(1-\beta \alpha)^{\ln (1-x) / \ln (1-\alpha)}
$$

A more convenient representation of this function is:

$$
(1-y)=C^{\ln (1-x)}
$$

in which

$$
C=(1-\beta \alpha)^{1 / \ln (1-\alpha)}
$$

a factor only depending on the relative area occupied by a single lesion $(\alpha)$ and the ratio between areas occupied by a virtual and a visual lesion $(\beta)$. When the logarithm of the left-hand term of equation 4 is taken and plotted against $\ln (1-x)$, a straight line with slope $\ln (C)$ is obtained:

$$
\ln (1-y)=\ln (C) \ln (1-x)
$$

Using equation 5 gives:

$$
\ln (C)=\ln (1-\beta \alpha) / \ln (1-\alpha)
$$

Because $\ln (1-z)$ is almost equal to $-z$, when $0<z<0.2$ (if $z$ is any variable),

$$
\ln (C) \approx-\beta \alpha /-\alpha=\beta
$$

when $\beta \alpha<0.2$. Equation 6 then reduces to

$$
\ln (1-y)=\beta \ln (1-x)
$$

This result implies that, as long as a virtual lesion does not occupy more than $20 \%$ of the area of a leaf, the relation between

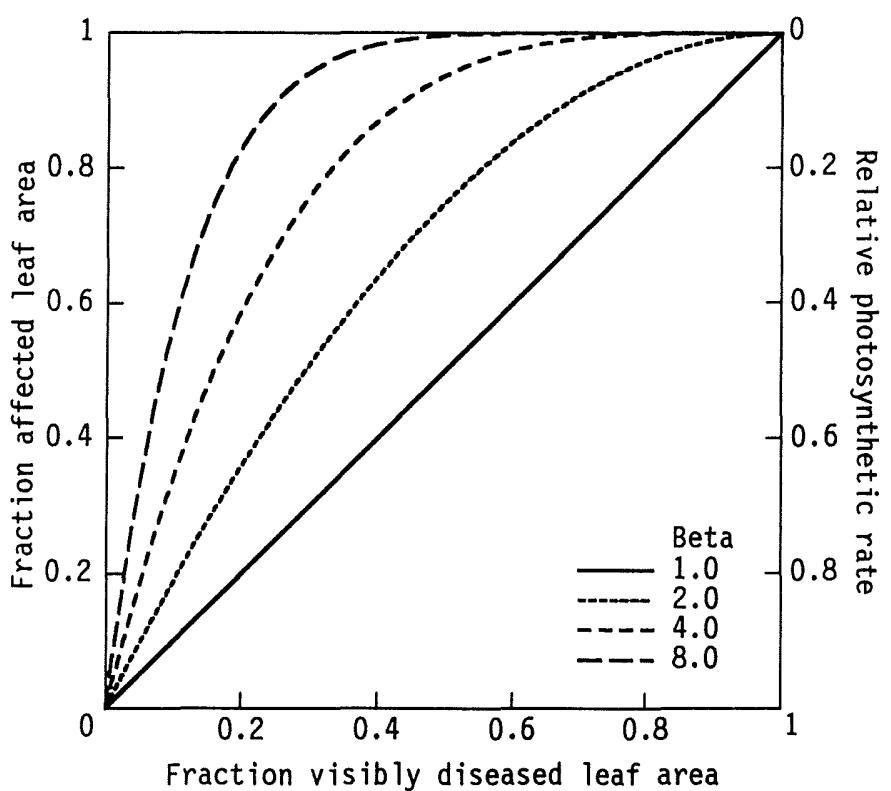

Fig. 1. The fraction affected leaf area $(y)$ and the relative photosynthetic rate $\left(P_{x} / P_{O}\right)$ of infected leaves in relation to the fraction visibly diseased leaf area $(x)$ for a leaf spot disease. A lesion is assumed to be part of a virtual lesion in which photosynthesis is negligible. The parameter $\beta$ expresses the ratio between virtual and visual lesion area. 
visibly diseased leaf area and affected leaf area is determined only by $\beta$. This also holds for a disease with variable lesion size, as long as $\beta$ is independent of lesion size. Combining equation 4 and equation 8 results in:

$$
y=1-\left(e^{\beta}\right)^{\ln (1-x)}=1-(1-x)^{\beta}
$$

In Figure 1, this equation is used to obtain the relation between the fraction visibly diseased leaf area $(x)$ and the fraction affected leaf area $(y)$, for various values of $\beta$. If leaf photosynthesis in the affected part of the leaf is impaired, the fraction of leaf area remaining virtually healthy will be expressed in the ratio of photosynthesis of the infected leaf and photosynthesis of a comparable healthy leaf:

$$
(1-y)=P_{x} / P_{O}
$$

in which $P_{x}=$ photosynthesis of a leaf with disease severity $x$, and $P_{O}=$ photosynthesis of a healthy leaf.

Combining equation 10 with equation 11 results in:

$$
P_{x} / P_{\mathrm{O}}=(1-x)^{\beta}
$$

in which the relative photosynthesis of an infected leaf is given in dependence of disease severity (Fig. 1). After the measured rates of photosynthesis of both healthy and diseased leaves were expressed as a fraction of the average leaf photosynthetic rate of healthy leaves, the parameter $\beta$ was determined by using nonlinear regression analysis. This analysis was performed by using the Genstat statistical package (7).

Independent sets of data used to explore utility of the model with other pathosystems. Two data sets on reduction of leaf photosynthesis in winter wheat due to leaf rust (19) and powdery mildew (18) were used to explore the utility of the model for other pathosystems. Spitters et al (19) measured leaf photosynthesis of the flag leaf on wheat cultivar Cappelle Desprez. The measurements were taken in the field using a portable leaf chamber gas analyzer similar to the one described previously. All measurements were made at light saturation provided by an incandescent lamp cooled by a fan. The percentage of remaining green leaf area on the infected leaf was determined. Measurements were taken in the whole range from 100 to $0 \%$ green leaf area. The results of individual measurements were presented in a graph and were used for this analysis.

Rabbinge et al (18) measured leaf photosynthesis on wheat cultivar Okapi at development stage DC 32 (decimal code, 26). Plants were grown in pots in a greenhouse and were inoculated 2.5 wk before measurement of photosynthesis. Equipment for routine measurements of photosynthesis as described by Louwerse and van Oorschot (12) was used to measure rates of leaf photosynthesis at different light intensities. The percentage of leaf area covered with mildew lesions was determined and ranged from 0 to $10 \%$. Average values of measured rates of photosynthesis, based on at least nine measurements, were presented in a table.

TABLE 1 . Net photosynthetic rate ( \pm standard error of the mean) of

\begin{tabular}{|c|c|c|c|c|c|c|}
\hline \multirow[b]{3}{*}{ Severity $^{\mathrm{a}}$} & \multirow[b]{3}{*}{ No. } & \multicolumn{2}{|l|}{ Field experiment } & \multicolumn{3}{|c|}{ Greenhouse experiment } \\
\hline & & \multicolumn{2}{|c|}{ Net photosynthesis } & \multirow[b]{2}{*}{ No. } & \multicolumn{2}{|c|}{ Net photosynthesis } \\
\hline & & $\left(\mathrm{kg} \mathrm{CO}_{2} / \mathrm{ha} / \mathrm{h}\right)$ & $P_{x} / P_{o}$ & & $\left(\mathrm{~kg} \mathrm{CO}_{2} / \mathrm{ha} / \mathrm{h}\right)$ & $P_{x} / P_{o}$ \\
\hline 0.00 & 38 & $44.1 \pm 0.5$ & 1 & 43 & & 1 \\
\hline $00-$ & 36 & & & 48 & & 0.95 \\
\hline $05-0.10$ & 19 & & 0.7 & 40 & & 0.75 \\
\hline & 11 & & 0. & 12 & & 0.64 \\
\hline $0.15-0.20$ & 3 & $24.1 \pm$ & 0.5 & 6 & \pm 1.7 & 0.45 \\
\hline$>0.20$ & 9 & $20.9 \pm 1.9$ & 0.47 & 8 & $14.8 \pm 2.0$ & 0.37 \\
\hline
\end{tabular}
rice leaves infected by Pyricularia oryzae, measured at high radiation levels, in field and greenhouse experiments

${ }^{\mathrm{a}}$ Leaves were grouped according to fraction of leaf area covered by blast lesions (disease severity).
The rate of photosynthesis at light saturation was used for this analysis.

\section{RESULTS AND DISCUSSION}

In both the field and greenhouse experiments, the reduction in leaf photosynthesis of infected leaves, expressed as a fraction of leaf photosynthetic rate of healthy leaves, surpassed disease severity (Table 1). The disease not only reduced the amount of green leaf area, but also affected the photosynthesis of the remaining green leaf tissue. The measurements of leaf photosynthesis from the field and greenhouse experiments are shown in Figures

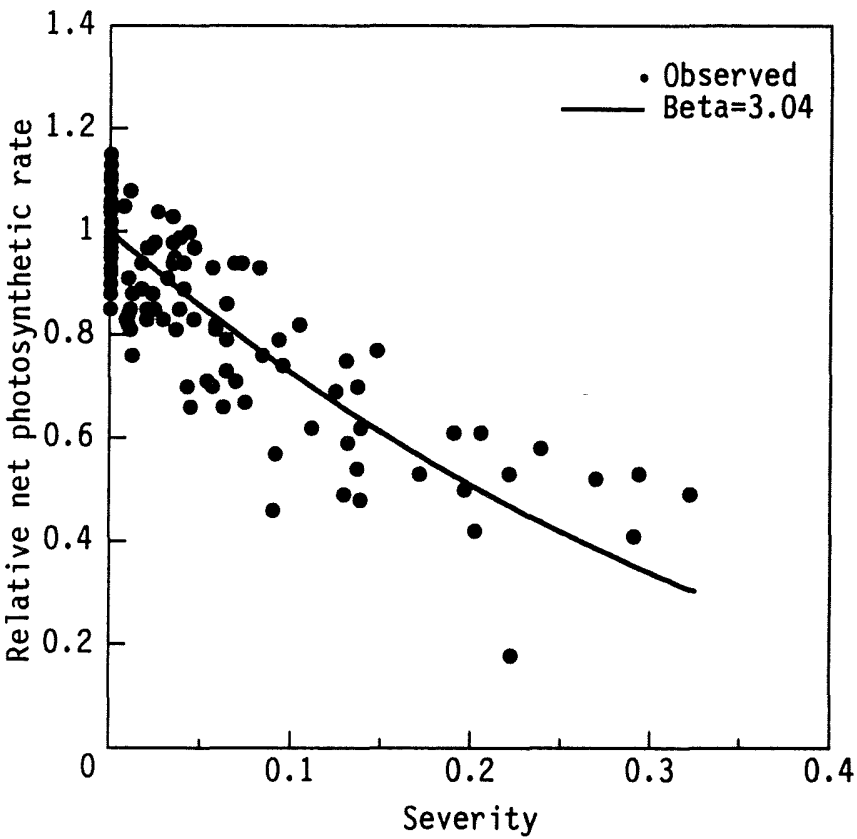

Fig. 2. Relative net photosynthetic rate of leaves infected by Pyricularia oryzae in relation to disease severity as measured in a field experiment. Equation 12 was used to describe the relative photosynthetic rate of an infected leaf in dependence of disease severity, using the best-fitting $\beta$ value.

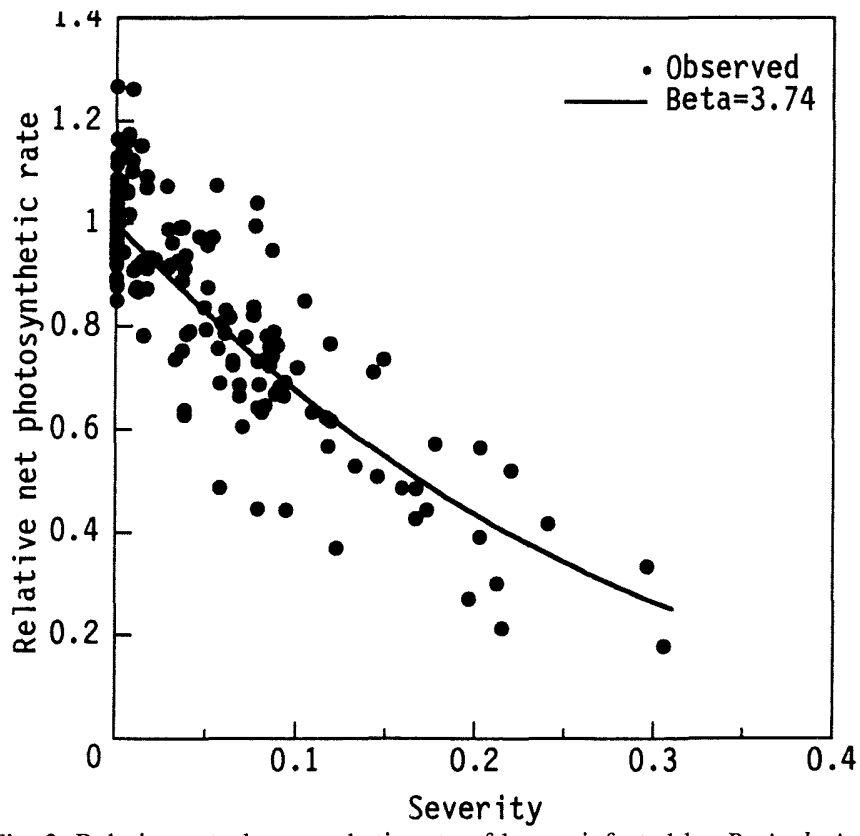

Fig. 3. Relative net photosynthetic rate of leaves infected by Pyricularia oryzae in relation to disease severity as measured in a greenhouse experiment. Equation 12 was used to describe the relative photosynthetic rate of an infected leaf in dependence of disease severity, using the best-fitting $\beta$ value. 
2 and 3, respectively. Net rate of photosynthesis was expressed relative to the average measured photosynthesis of healthy leaves. Equation 12 was used to describe the relation between disease severity and leaf photosynthesis. In both experiments, a $\beta$ value between 3 and 4 gave the best description of leaf photosynthetic rate in dependence of severity. The percentage of variance accounted for by these curves was $71 \%$ in Figure 2 and $74 \%$ in Figure 3. Based on these percentages, together with the homogeneous distribution of residual variance around the fitted curves, equation 12 gave an adequate quantitative description of the effect of leaf blast on photosynthesis of rice leaves. This demonstrates that the effect of leaf blast on leaf photosynthetic rate of rice leaves, for the measured range of severities, can be expressed with a single parameter.

The same equation gave a good description of the relation between leaf photosynthetic rate and disease severity reported for other pathosystems (Table 2). The reduction in leaf photosynthetic rate due to leaf rust in wheat was characterized with a $\beta$ value that did not differ significantly from 1 . This is in accordance with the conclusion drawn by Spitters et al (19) that after leaf rust infection the photosynthetic capacity of the remaining green surface is not affected. The $\beta$ value for powdery mildew in wheat expressed clearly that the pathogen's effect on leaf photosynthetic rate of the host is much stronger than in the case of leaf blast in rice.

Although the measurements of leaf photosynthesis demonstrate that leaf blast of rice reduced the photosynthesis of the remaining green leaf tissue, the measurements neither clarify the mechanism responsible for this reduction nor indicate the location of this effect. The strongest reduction in leaf photosynthesis would be expected in the surroundings of the visual lesion. This situation could result from production and secretion of a toxic compound, which then diffuses to the surrounding area of the lesion. Toxins produced by $P$. oryzae have been isolated from diseased plant tissues (20), but effects of the isolated toxins on leaf photosynthesis have not been reported. Yoshii (25) reported that cell walls and cell inclusions in the central part of the lesion of $P$. oryzae disintegrate. As a result of this disintegration, the transport of either water or assimilates or both may be impaired, and consequently the leaf tissue situated near the lesion may be affected. A reduced relative water content may affect photosynthetic rate either indirectly by closure of the stomata or directly through an inhibition at the chloroplast level (10). Reduced translocation of assimilates may result in accumulation of carbohydrates (14). For various plant species, a strong negative relation between carbohydrate accumulation and net uptake of $\mathrm{CO}_{2}$ was observed, indicating feedback inhibition of photosynthesis $(1,4,11,21)$.

Waggoner and Berger (24) argued that radiation absorbed by healthy leaf area is adequate to explain yield in most pathosystems. Following their reasoning, one may wonder whether leaf photosynthesis measurements contribute to a quantitative understanding of disease-induced yield loss. Johnson (8), however, distinguished two major effects of diseases on crop growth: a reduction in the solar radiation interception (RI) by green leaf area, and a reduction in the radiation use efficiency (RUE). The present study indicates that leaf blast in rice is an example of

TABLE 2. Characterization of the reduction in leaf photosynthetic rate of the host due to the presence of a pathogen, for various pathosystems, using the estimated parameter $\beta$

\begin{tabular}{lccl}
\hline Pathosystem $^{\text {a }}$ & $\beta$ & $r^{2}$ & Source \\
\hline $\begin{array}{l}\text { Puccinia recondita- } \\
\text { winter wheat }\end{array}$ & $1.26 \pm 0.17^{b}$ & 0.77 & Spitters et al (19) \\
$\begin{array}{l}\text { Pyricularia oryzae- } \\
\text { rice }\end{array}$ & $3.04 \pm 0.18$ & 0.71 & Field experiment \\
$\begin{array}{l}\text { Erisyphe graminis- } \\
\text { winter wheat }\end{array}$ & $3.74 \pm 0.19$ & 0.74 & Greenhouse experiment \\
& $8.74 \pm 1.70$ & 0.84 & Rabbinge et al (18) \\
\hline
\end{tabular}

${ }^{a}$ The data refer to measurements at high light intensities.

${ }^{\mathrm{b}} \mathrm{Standard}$ error of estimated $\beta$. a host-pathogen combination in which both effects occur. For an analysis of crop growth in terms of RI and RUE, only the time course of green leaf area is needed. Determination of disease severity and its effect on photosynthesis is not a prerequisite. However, for purposes of crop protection rather than analyzing in retrospect, the construction of damage relations is the ultimate aim. This requires the relation between RUE of green leaf tissue and disease severity. Next to the level of disease, other factors will have their impact on an aggregated parameter as RUE, preventing the existence of a general relationship between disease severity and RUE. Experimental determination of this relation for all possible combinations of crop status and environmental factors will take a lot of effort. Introduction of the measured leaf photosynthesis damage relationship into a process-level crop growth simulator, as proposed by Boote et al (2), provides quantitative insight into the consequences for crop growth and grain yield. A well-documented growth model for rice, in which the calculation of canopy photosynthesis is based on leaf photosynthetic rate of various leaf layers, has been developed recently (MACROS, 17). Such a model enables the establishment of the relation between RUE of green leaf tissue and disease severity for various conditions. Summarizing the outcomes in graphs or simple regression equations will be useful for the application of the type of model discussed by Johnson (8). In my opinion, using simple models, and parameterizing them with the results from more detailed models, will enhance the impact of both model types on the construction of damage relations.

\section{LITERATURE CITED}

1. Azcon-Bieto, J. 1983. Inhibition of photosynthesis by carbohydrates in wheat leaves. Plant Physiol. 73:681-686.

2. Boote, K. J., Jones, J. W., Mishoe, J. W., and Berger, R. D. 1983. Coupling pests to crop growth simulators to predict yield reductions. Phytopathology 73:1581-1587.

3. Burrell, M. M., and Rees, T. 1974. Carbohydrate metabolism of rice leaves infected by Pyricularia oryzae. Physiol. Plant Pathol. 4:489496.

4. Chatterton, N. J. 1973. Product inhibition of photosynthesis in alfalfa leaves as related to specific leaf weight. Crop Sci. 13:284-285.

5. Ephrath, J. E., Shteinberg, D., Drieshpoun, J., Dinoor, A., and Marani, A. 1989. Alternaria alternata in cotton (Gossypium hirsutum) cv. Acala: Effects on gas exchange, yield components and yield accumulation. Neth. J. Plant Pathol. 95:157-166.

6. Goto, K. 1965. Estimating losses from rice blast in Japan. Pages 195-202 in: The Rice Blast Disease. Proceedings of a Symposium at IRRI, 1963. Johns Hopkins University Press, Baltimore, MD.

7. Gower, J. C., Tunnicliffe Wilson, G., and Paterson, L. J. 1987. Genstat 5 Reference Manual. Clarendon Press, Oxford. 749 pp.

8. Johnson, K. B. 1987. Defoliation, disease and growth: A reply. Phytopathology 77:1495-1497.

9. Justesen, J. H., and Tammes, P. M. L. 1960. Studies on yield losses. I. The self-limiting effect of injurious or competitive organisms on crop yield. Tijdschr. Plantenziekten 66:281-287.

10. Kaiser, W. M. 1987. Effects of water deficit on photosynthetic capacity. Physiol. Plant. 71:142-149.

11. Ku, S. B., Edwards, G. E., and Smith, D. 1978. Photosynthesis and nonstructural carbohydrate concentration in leaf blades of Panicum virgatum as affected by night temperature. Can. J. Bot. 56:63-68.

12. Louwerse, W., and van Oorschot, J. L. P. 1969. An assembly for routine measurements of photosynthesis, respiration and transpiration of intact plants under controlled conditions. Photosynthetica 3:305315 .

13. Mackill, A. O., and Bonman, J. M. 1986. New hosts of Pyricularia oryzae. Plant Dis. 70:125-127.

14. Neales, T. F., and Incoll, L. D. 1968. The control of leaf photosynthesis rate by the level of assimilate concentration in the leaf: A review of the hypothesis. Bot. Rev. 34:107-125.

15. Ou, S. H. 1985. Rice Diseases. 2nd ed. Commonwealth Mycological Institute, Kew, Surrey, England. 380 pp.

16. Padhi, B., Chakrabarti, N. K., and Nayak, S. K. 1978. Effect of blast on the photosynthetic activity of rice seedlings. Biol. Plant. 20:418-420.

17. Penning de Vries, F. W. T., Jansen, D. M., ten Berge, H. F. M., and Bakema, A. 1989. Simulation of Ecophysiological Processes of Growth in Several Annual Crops. PUDOC, Wageningen, The Netherlands. 271 pp. 
18. Rabbinge, R., Jorritsma, I. T. M., and Schans, J. 1985. Damage components of powdery mildew in winter wheat. Neth. J. Plant Pathol. 91:235-247.

19. Spitters, C. J. T., van Roermund, H. J. W., van Nassau, H. G. M. G. Schepers, J., and Mesdag, J. 1990. Genetic variation in partial resistance to leaf rust in winter wheat: Disease progress, foliage senescence and yield reduction. Neth. J. Plant Pathol, 96:3-15.

20. Tamari, K., and Kaji, J. 1954. Biochemical studies of the blast fungus (Pyricularia oryzae Cav.), the causative fungus of the blast disease of rice plants. I. Studies on the toxins produced by blast fungus. J. Agric. Chem. Soc. Jpn. 28:254-258.

21. Upmeyer, D. J., and Koller, H. R. 1973. Diurnal trends in net photosynthetic rate and carbohydrate levels of soybean leaves. Plant
Physiol. 51:871-874.

22. van Oijen, M. 1990 . Photosynthesis is not impaired in healthy tissue of blighted potato plants. Neth. J. Plant Pathol. 96:55-63.

23. von Caemmerer, S., and Farquhar, G. D. 1981. Some relationships between the biochemistry of photosynthesis and the gas exchange of leaves. Planta 153:376-387.

24. Waggoner, P. E., and Berger, R. D. 1987. Defoliation, disease, and growth. Phytopathology 77:393-398.

25. Yoshii, H. 1937. Pathological studies of rice blast caused by Pyricularia oryzae. III. Pathohistological observations of diseased plants. Ann. Phytopathol. Soc. Jpn. 7:289-304.

26. Zadoks, J. C., Chang, T. T., and Konzak, C. F. 1974. A decimal code for the growth stages of cereals. Weed Res. 14:415-421. 
